\title{
Weak mixing in general semiflows implies multi-sensitivity, but not thick sensitivity
}

\section{Alica Miller}

Department of Mathematics, University of Louisville, USA.

\begin{abstract}
It was proved by Wang et al. [Wang, J. Yin, Q. Yan, J. Nonlinear Sci. Appl., 9 (2016), 989-997] that any weakly mixing semiflow on a compact metric space, whose all transition maps are surjective, is thickly sensitive. We consider what happens if we do not have the assumptions of compactness and surjectivity. We prove that even in that case any weakly mixing semiflow is multi-sensitive, and that, however, it does not have to be thickly sensitive.
\end{abstract}

Keywords: Weak mixing, sensitivity, multi-sensitivity, thick sensitivity, semi-flow.

2010 MSC: 37B05, 54H20, 37D45, 37B20, 37B99.

(C)2019 All rights reserved.

\section{Introduction}

Research on relations between the mixing properties and sensitivity in semiflows has a long history. We will mention some recent papers. In 2004 it was proved in [2] by He et al. that weak mixing implies sensitivity in the context of Ergodic Theory. In 2006 it was proved in [4] by Lardjane that strong mixing implies sensitivity for topological semiflows. In 2007 in [7] Moothathu introduced the notion of multisensitivity for semiflows and mentioned that (1) every weakly mixing compact cascade is multi-sensitive and that (2) every multi-sensitive compact cascade is thickly sensitive. Note that in compact cascades every transition map is surjective. Indeed, if $(X, f)$ is a compact cascade, then $f$ is surjective, otherwise nothing from $X$ would be mapped to the open set $X \backslash f(X)$ by any transition map. In 2016 it was proved in [8] by Wang et al. that any semiflow on a compact metric space (with an arbitrary acting topological monoid) whose every transition map is surjective is thickly sensitive, thus generalizing Moothathu's comments. In this paper we consider what happens if we do not have the assumptions of compactness of the phase space and surjectivity of the transition maps. We prove that the semiflow is still multi-sensitive (Theorem 2.3 below) and that, however, it does not have to be thickly sensitive (Theorem 2.4 below). We thus generalized Moothathu's first comment to the case of general semiflows and showed that his second comment does not hold in general semiflows. Let us also mention that multi-sensitivity for continuous semiflows was discussed in [5].

Email address: alica.miller@louisville.edu (Alica Miller)

doi: $10.22436 /$ jnsa.012.02.05

Received: 2017-12-31 Revised: 2018-08-07 Accepted: 2018-08-23 
We now give some definitions. In this paper $T$ will always denote a noncompact abelian topological monoid, written additively, whose identity element is 0 . For example: $\left(\mathbb{N}_{0},+\right)$, where $\mathbb{N}_{0}=\{0,1,2, \ldots\}$, $\left(\mathbb{N}_{0}^{2},+\right),(\mathbb{Z},+),\left(\mathbb{R}_{+},+\right)$, where $\mathbb{R}_{+}=[0, \infty),\left(\mathbb{R}_{+}^{2},+\right),(\mathbb{R},+)$, etc.

A subset $A$ of $T$ is called thick if for every compact subset $K$ of $T$ there is a $t \in T$ such that $t+K \subseteq A$.

If $(X, d)$ is a metric space, $x \in X$ and $r>0$, the open ball with center $x$ and radius $r$ is denoted by $B(x, r)$. It consist of all points $y \in X$ such that $d(x, y)<r$. The closed ball with center $x$ and radius $r$ is denoted by $B^{-}(x, r)$. It consist of all points $y \in X$ such that $d(x, y) \leqslant r$. It is a closed subset of $X$.

A jointly continuous monoid action $\pi: T \times X \rightarrow X$ of a topological monoid $T$ on a metric space $(X, d)$ is called a semiflow and denoted by $(T, X, \pi)$ or by $(T, X)$. The element $\pi(t, x)$ will be denoted by $t . x$ or $t x$, so that the defining conditions for a monoid action have the form

$$
\text { s. }(\mathrm{t} \cdot \mathrm{x})=(\mathrm{s}+\mathrm{t}) \cdot \mathrm{x}, \quad 0 \cdot \mathrm{x}=\mathrm{x},
$$

for any $s, t \in T$ and $x \in X$. The maps $\pi_{t}: X \rightarrow X, x \mapsto t x$ are called the transition maps. For any $x \in X$ the set $T x=\{t x \mid t \in T\}$ is called the orbit of $x$. The metric space $X$ is called the phase space.

An $\mathbb{N}_{0}$-semiflow $\left(\mathbb{N}_{0}, X, \pi\right)$ is called a cascade. It is completely determined by the transition map $f:=\pi_{1}$ since $\pi_{n}=f^{n}$ for every $n \in \mathbb{N}_{0}$. It is also denoted by $(X, f)$. For a similar reason the $\mathbb{Z}$-flows are called cascades.

For any two open sets $U, V$ of the phase space $X$ in a semiflow $(T, X)$ we denote $D(U, V)=\{t \in$ $\mathrm{T} \mid \mathrm{tU} \cap \mathrm{V} \neq \emptyset\}$.

A semiflow $(T, X)$ is called topologically transitive if for any nonempty open subsets $\mathrm{U}, \mathrm{V}$ of $\mathrm{X}$ there is a $t \in T$ such that $t U \cap V \neq \emptyset$. A semiflow $(T, X)$ is called weakly mixing (WM) if for any four nonempty open subsets $U_{1}, V_{1}, U_{2}, V_{2}$ of $X$ there is a $t \in T$ such that $t U_{1} \cap V_{1} \neq \emptyset$ and $t U_{2} \cap V_{2} \neq \emptyset$. A semiflow $(T, X)$ is called strongly mixing if for any two nonempty open subsets $\mathrm{U}, \mathrm{V}$ of $\mathrm{X}$ there is a compact subset $\mathrm{K}$ of $\mathrm{T}$ such that for every $t \in T \backslash K, t U \cap V \neq \emptyset$. Every strongly mixing semiflow is weakly mixing. A semiflow $(T, X)$ is called sensitive if there is a number $c>0$ (called a sensitivity constant) such that for any nonempty open set $\mathrm{U} \subset \mathrm{X}$ there are two points $\mathrm{x}, \mathrm{y} \in \mathrm{U}$ and $\mathrm{t} \in \mathrm{T}$ with $\mathrm{d}(\mathrm{t} x, \mathrm{t} \mathrm{y})>\mathrm{c}$.

The paper is self-contained, i.e., all the notions used in the paper are defined in it. The reader can also consult the papers $[3,6]$ for additional information.

\section{Sensitivity of general weakly mixing semiflows}

The following lemma is well-known, but there is no good reference for the proof for general semiflows. Our proof is similar to the proof in [1].

Lemma 2.1. Let $(\mathrm{T}, \mathrm{X})$ be a weakly mixing semiflow and $\mathrm{U}_{1}, \ldots, \mathrm{U}_{\mathrm{n}}, \mathrm{V}_{1}, \ldots, \mathrm{V}_{\mathrm{n}}$ nonempty open subsets of $\mathrm{X}$ $(\mathrm{n} \geqslant 1)$. Then there is $a \mathrm{t} \in \mathrm{T}$ such that $\mathrm{tu}_{i} \cap \mathrm{V}_{\mathrm{i}} \neq \emptyset$ for all $\mathrm{i}=1, \ldots, \mathrm{n}$.

Proof. The proof is by induction on $n$. This is clearly true for $n=1$ and $n=2$ by the definition of weak mixing. Suppose the statement is true for $n \geqslant 2$ pairs od sets. Consider $n+1$ pairs of sets $U_{i}, V_{i}$, $\mathrm{I}=1,2, \ldots, \mathrm{n}+1$. Let

$$
\mathrm{t} \in \mathrm{D}\left(\mathrm{U}_{1}, \mathrm{~V}_{1}\right) \cap \mathrm{D}\left(\mathrm{U}_{2}, \mathrm{~V}_{2}\right)
$$

and

$$
s \in D\left(U_{1} \cap t^{-1} V_{1}, u_{2} \cap t^{-1} V_{2}\right) \cap D\left(U_{3}, V_{3}\right) \cap \cdots \cap D\left(u_{n+1}, V_{n+1}\right) .
$$

Then $\left(U_{1} \cap t^{-1} V_{1}\right) \cap s^{-1}\left(U_{2} \cap t^{-1} V_{2}\right) \neq \emptyset$, hence $s \in D\left(U_{1}, V_{1}\right) \cap D\left(U_{2}, V_{2}\right)$. Hence $s \in D\left(U_{1}, V_{1}\right) \cap$ $\mathrm{D}\left(\mathrm{U}_{2}, \mathrm{~V}_{2}\right) \cap \cdots \cap \mathrm{D}\left(\mathrm{U}_{\mathrm{n}+1}, \mathrm{~V}_{\mathrm{n}+1}\right)$.

Definition 2.2. A semiflow $(T, X)$ is multi-sensitive if there is a real number $c>0$ such that for any integer $m \geqslant 1$ and any nonempty open subsets $U_{1}, U_{2}, \ldots, U_{m}$ of $X$ there is a $t \in T$ with the following property: for every $i \in\{1,2, \ldots, m\}$ there are $x_{i}$ and $y_{i}$ in $u_{i}$ such that $d\left(t x_{i}, t y_{i}\right) \geqslant c$. 
It is important to mention that in the next theorem we do not assume neither that $X$ is compact nor that the transition maps have dense images.

Theorem 2.3. Every weakly mixing semiflow on a metric space consisting of more than one point is multi-sensitive.

Proof. Let $\Delta=\operatorname{diam}(X)>0$. Then for any ball $B(x, \Delta / 3), x \in X$, we have $X \backslash B(x, \Delta / 3) \neq \emptyset$. We will show that $(T, X)$ is multi-sensitive with sensitivity constant $c=\Delta / 12$. Let $m \geqslant 1$ be an integer and let $\mathrm{U}_{1}, \mathrm{U}_{2}, \ldots, \mathrm{U}_{\mathrm{m}}$ be nonempty open subsets of $X$. Let $\mathrm{x}_{i} \in \mathrm{U}_{i}(i=1,2, \ldots, \mathrm{m})$. For each $i=1,2, \ldots, \mathrm{m}$ let $\mathrm{B}_{i}=\mathrm{B}\left(\mathrm{x}_{i}, r_{i}\right)$, where $r_{i}<\Delta / 12$ is such that $\mathrm{B}_{i} \subseteq \mathrm{U}_{i}$. Let also $C_{i}^{-}=\mathrm{B}^{-}\left(\mathrm{x}_{i}, \Delta / 6\right)$. Then each $V_{i}=X \backslash C_{i}^{-}$ is a nonempty open subset of $X$. Note that for any $a \in B_{i}$ and $b \in V_{i}$,

$$
d(a, b) \geqslant d\left(b, x_{i}\right)-d\left(a, x_{i}\right)>\Delta / 6-\Delta / 12=\Delta / 12 .
$$

By Lemma 2.1 there is a $t \in \mathrm{T}$ such that the following $2 \mathrm{~m}$ relations hold all at the same time:

$$
t B_{i} \cap B_{i} \neq \emptyset \quad \text { for } i=1,2, \ldots, m, \quad t B_{i} \cap V_{i} \neq \emptyset \quad \text { for } i=1,2, \ldots, m .
$$

Let $y_{i}, z_{i} \in B_{i} \subseteq U_{i}(i=1,2, \ldots, m)$ be such that

$$
t y_{i} \in B_{i} \quad \text { for } i=1,2, \ldots, m, \quad t z_{i} \in V_{i} \quad \text { for } i=1,2, \ldots, m .
$$

Then (as we noticed earlier for any points $a, b) d\left(t y y_{i}, t z_{i}\right)>\Delta / 12 \quad(i=1,2, \ldots, m)$. The theorem is proved.

The following is Theorem 4.11 from [8].

Theorem 2.4 ([8]). Let $(\mathrm{T}, \mathrm{X})$ be a semiflow on a compact metric space $\mathrm{X}$ whose all transition maps are surjective. If $(\mathrm{T}, \mathrm{X})$ is weakly mixing, then it is thickly sensitive.

We consider what happens if we do not have the assumptions that $X$ is compact and all transition maps surjective. By our previous theorem we know that $(T, X)$ is still multi-sensitive, however, in the next theorem we show that we cannot reach the conclusion that $(T, X)$ has to be thickly sensitive, like in the theorem of Wang et al.. So the assumptions that they have in their theorem are essential.

Theorem 2.5. There exists a weakly mixing semiflow which is not thickly sensitive.

Proof. Let $\mathbb{T}$ be a one-dimensional torus $\mathbb{R} / \mathbb{Z}$, i.e., $\mathbb{T}=[0,1)$ with the metric $d(x, y)=\min \{|x-y|, 1-$ $|x-y|\}$. Define a continuous function $f: \mathbb{T} \rightarrow \mathbb{T}$ by $f(x)=2 x(\bmod 1)$ for every $x \in \mathbb{T}$. A point $x \in \mathbb{T}$ in the cascade $(\mathbb{T}, f)$ is said to be eventually fixed if there is an $n \geqslant 0$ such that $f^{n}(x)=0$. The set of all eventually fixed points is $X=\left\{k / 2^{n} \mid k, n \geqslant 0\right.$ integers, $\left.k<2^{n}\right\}$, which is a dense subset of $\mathbb{T}$. Note that $f(X) \subseteq X$, so that we can consider the restricted semiflow $(X, f)$. Each point in this semiflow has a finite orbit whose last term is 0 . The point 0 is the only fixed point. We now show that $(X, f)$ is weakly mixing. Let $\mathrm{U}_{1}=\left\{x \in X \mid \mathrm{a}_{1}<x<\mathrm{b}_{1}\right\}, \mathrm{V}_{1}=\left\{x \in X \mid \mathrm{c}_{1}<x<\mathrm{d}_{1}\right\}, \mathrm{U}_{2}=\left\{x \in X \mid \mathrm{a}_{2}<x<\mathrm{b}_{2}\right\}$, and $V_{2}=\left\{x \in X \mid c_{2}<x<d_{2}\right\}$ be four open subsets of $X$, where $a_{1}, b_{1}, c_{1}, d_{1}, a_{2}, b_{2}, c_{2}, d_{2}$ are real numbers from $[0,1)$. Let $x_{0}=k / 2^{m}$ be an element of $V_{1}$ with $k$ an odd number. Then

$$
X_{1}:=f^{-1}\left(x_{0}\right)=\left\{\frac{k}{2^{m+1}}, \frac{k}{2^{m+1}}+\frac{1}{2}\right\}=\left\{x_{1,0}=\frac{k}{2^{m+1}}, x_{1,1}=\frac{k+2^{m}}{2^{m+1}}\right\} .
$$

The distance between the two elements of $X_{1}$ is $1 / 2^{1}$. Now

$$
\begin{aligned}
X_{2}:=f^{-1}\left(X_{1}\right) & =\left\{\frac{k}{2^{m+2}}, \frac{k}{2^{m+2}}+\frac{1}{4}, \frac{k}{2^{m+2}}+\frac{2}{4}, \frac{k}{2^{m+2}}+\frac{3}{4}\right\} \\
& =\left\{x_{2,0}=\frac{k}{2^{m+2}}, x_{2,1}=\frac{k+2^{m}}{2^{m+2}}, x_{2,2}=\frac{k+2 \cdot 2^{m}}{2^{m+2}}, x_{2,3}=\frac{k+3 \cdot 2^{m}}{2^{m+2}}\right\} .
\end{aligned}
$$


The distance between the neighboring elements of $X_{2}$ is $\frac{1}{2^{2}}$, including the distance between $x_{2,0}$ and $x_{2,3}$. The sets $X_{3}:=f^{-1}\left(X_{2}\right), X_{4}:=f^{-1}\left(X_{3}\right), \ldots$ have analogous properties. If $r_{1} \in \mathbb{N}$ is such that $b_{1}-a_{1}>\frac{1}{2^{r_{1}}}$, then for any $r \geqslant r_{1}$ an element $x_{r, i}$ of $X_{r}$ is in $U_{1}$. Hence $f^{r}\left(x_{r, i}\right)=x_{0}$, so that $f^{r}\left(u_{1}\right) \cap V_{1} \neq \emptyset$.

Let now $y_{0}=l / 2^{n}$ be an element of $V_{2}$ with $l$ an odd number. In the same way as before we form for $y_{0}$ the sets $Y_{1}, Y_{2}, \ldots$ (analogous to the sets $X_{1}, X_{2}, \ldots$ for $x_{0}$ ). We conclude that if $r_{2} \in \mathbb{N}$ is such that $b_{2}-a_{2}>\frac{1}{2^{r_{2}}}$, then for any $r \geqslant r_{2}$ an element $y_{r, j}$ of $Y_{r}$ is in $u_{2}$. Hence $f^{r}\left(y_{r, j}\right)=y_{0}$, so that $f^{r}\left(U_{2}\right) \cap V_{2} \neq \emptyset$. Taking $r \geqslant \max \left\{r_{1}, r_{2}\right\}$ we have both $f^{r}\left(U_{1}\right) \cap V_{1} \neq \emptyset$ and $f^{r}\left(U_{2}\right) \cap V_{2} \neq \emptyset$, so that we can conclude that $(X, f)$ is weakly mixing.

Let now $T_{1}=\{0,1\}$ be a discrete monoid with the operation $0+0=0,1+0=0+1=1+1=1$. Let $\mathrm{T}=\mathbb{N}_{0} \times \mathrm{T}_{1}=\left\{(\mathrm{n}, \mathrm{t}) \mid \mathrm{n} \in \mathbb{N}_{0}, \mathrm{t} \in \mathrm{T}_{1}\right\}$ be the product monoid of the discrete monoids $\mathbb{N}_{0}$ and $\mathrm{T}_{1}$ (with componentwise addition). Define a monoid action of $\mathrm{T}$ on $\mathrm{X}$ by

$$
(n, t) \cdot x= \begin{cases}f^{n}(x), & \text { if } t=0 \\ 0, & \text { if } t=1\end{cases}
$$

It is easy to verify that this is indeed a monoid action. All transition maps are clearly continuous, so we have a topological semi-flow $(T, X)$. This semiflow is weakly mixing since $(X, f)$ is weakly mixing. Note that none of the transition maps $x \rightarrow(n \cdot 1)$. $x$ from $X$ to $X\left(n \in \mathbb{N}_{0}\right)$ has a dense image as they map all the points of $X$ to 0 .

We now show that $(T, X)$ is not thickly sensitive. Let $U$ be any open set in $X$ and let $c$ be any positive number. The set $D(U, c)$ does not contain any translate $(n, t)+K$ of the compact $K=\{(0,1)\}$, i.e., $D(U, c)$ does not contain any element $(n, 1) \in T$. Indeed, for any two points $x, y \in U$ we have $(n, 1) \cdot x=(n, 1) \cdot y=$ $0<\mathrm{c}$. Thus $\mathrm{D}(\mathrm{U}, \mathrm{c})$ is not thick.

\section{Conclusion}

We considered a weakly mixing semiflow whose phase space is not necessarily compact and whose transition maps do not necessarily have dense images. We proved that the semiflow is still multi-sensitive (Theorem 2.3 above) and that, however, it does not have to be thickly sensitive (Theorem 2.3 above) like in the case when we have those assumptions. It would be interesting to investigate the case when the phase space is not necessarily compact, but the transition maps do have dense images.

\section{Acknowledgment}

The author would like to acknowledge partial support by the National Science Foundation grant DMS 1405815.

\section{References}

[1] G. Cairns, A. Kolganova, A. Nielsen, Topological transitivity and mixing notions for group actions, Rocky Mount. J. Math., 37 (2007), 371-397. 2

[2] L. He, X. Yan, L. Wang, Weak-mixing implies sensitive dependence, J. Math. Anal. Appl., 299 (2004), 300-304. 1

[3] E. Kontorovich, M. Megrelishvili, A note on sensitivity of semigroup actions, Semigroup Forum, 76 (2008), $133-141$. 1

[4] S. Lardjane, On some stochastic properties in Devaney's chaos, Chaos. Solitons Fractals, 28 (2006), 668-672. 1

[5] R. Li, T. Lu, Y. Zhao, H. Wang, H. Liang, Multi-sensitivity, syndetical sensitivity and the asymptotic average-shadowing property for continuous semi-flows, J. Nonlinear Sci. Appl., 10 (2017), 4940-4953. 1

[6] A. Miller, Envelopes of syndetic subsemigroups of the acting topological semigroup in a semiflow, Topology Appl., 158 (2011), 291-297. 1

[7] T. K. S. Moothathu, Stronger forms of sensitivity for dynamical systems, Nonlinearity, 20 (2007), 2115-2126. 1

[8] T. Wang, J. Yin, Q. Yan, The sufficient conditions for dynamical systems of semigroup actions to have some stronger forms of sensitivities, J. Nonlinear Sci. Appl., 9 (2016), 989-997. 1, 2, 2.4 